\title{
Mixed biexcitons in single quantum wells
}

\section{Wagner, Hans Peter; Langbein, Wolfgang Werner; Hvam, Jørn Märcher}

\section{Published in:}

Physical Review B

Link to article, DOI:

10.1103/PhysRevB.59.4584

Publication date:

1999

Document Version

Publisher's PDF, also known as Version of record

Link back to DTU Orbit

Citation (APA):

Wagner, H. P., Langbein, W. W., \& Hvam, J. M. (1999). Mixed biexcitons in single quantum wells. Physical Review B, 59(7), 4584-4587. https://doi.org/10.1103/PhysRevB.59.4584

\section{General rights}

Copyright and moral rights for the publications made accessible in the public portal are retained by the authors and/or other copyright owners and it is a condition of accessing publications that users recognise and abide by the legal requirements associated with these rights.

- Users may download and print one copy of any publication from the public portal for the purpose of private study or research.

- You may not further distribute the material or use it for any profit-making activity or commercial gain

- You may freely distribute the URL identifying the publication in the public portal

If you believe that this document breaches copyright please contact us providing details, and we will remove access to the work immediately and investigate your claim. 


\title{
Mixed biexcitons in single quantum wells
}

\author{
H. P. Wagner* \\ TU Chemnitz, Institut für Physik, D-09107 Chemnitz, Germany \\ W. Langbein and J. M. Hvam \\ Mikroelektronik Centret, The Technical University of Denmark, 2800 Lyngby, Denmark
}

(Received 8 September 1998)

\begin{abstract}
Biexcitonic complexes in a $\mathrm{ZnSe}$ single quantum well are investigated by spectrally resolved four-wave mixing (FWM). The formation of heavy-heavy-hole $X X_{h}$ and of mixed heavy-light-hole $X X_{m}$ biexcitons showing binding energies of $\Delta_{h}=4.8 \mathrm{meV}$ and $\Delta_{m}=2.8 \mathrm{meV}$ is identified by polarization selection rules. The coherent dynamics of the FWM response and the observed FWM intensity ratio between the $X X_{h}$ and $X X_{m}$ biexciton-induced nonlinear signals are in agreement with the solution of an extended optical Bloch equation. [S0163-1829(99)01508-8]
\end{abstract}

The formation of biexcitons in low-dimensional semiconductor structures has attracted attention in recent years since biexcitonic effects that lead to an enhanced optical nonlinearity are important for all-optical and electro-optical device applications. These effects are particularly interesting in II-VI quantum-well (QW) structures because of their large exciton oscillator strength and high biexciton binding energies compared to III-V based structures. Most of the biexcitonic effects in II-VI structures have been investigated using high-excitation photoluminescence. ${ }^{1-4}$ Since a biexciton can be directly excited by two-photon excitation, the nonlinear optical technique of degenerate four-wave mixing (FWM) provides a powerful tool to study coherent biexcitonic phenomena. $^{4-7}$ So far, these studies consider mainly the biexciton formation from two heavy-hole excitons $\left(X_{h}\right)$. Biexcitons involving light-hole excitons $\left(X_{l}\right)$ have been observed, ${ }^{8}$ but no distinction between mixed and pure lighthole biexcitons has been made.

In this Brief Report we report on the formation of heavylight-hole biexcitons, denoted as mixed biexcitons $\left(X X_{m}\right)$, observed in FWM. We identify their contribution to the FWM signal using the polarization selection rules of the FWM response.

The investigated $\mathrm{ZnSe}$ single QW structure was pseudomorphically grown on (001) GaAs by molecular-beam epitaxy. The active $\mathrm{ZnSe}$ layer of $10-\mathrm{nm}$ thickness is sandwiched between two 25-nm-thick $\mathrm{Zn}_{0.9} \mathrm{Mg}_{0.1}$ Se barriers, defining a type-I QW. A detailed description of the growth and a sample characterization is given in Ref. 9. A frequency-doubled, mode-locked Ti-sapphire laser was used to excite the FWM, providing $100 \mathrm{fs}$ pulses of a spectral width of $22 \mathrm{meV}$ and a repetition rate of $76 \mathrm{MHz}$. We performed two-pulse degenerate FWM experiments in reflection geometry. The polarizations of the two incident pulses with the directions $\mathbf{k}_{1}$ and $\mathbf{k}_{2}$ and the mutual delay time $\tau$ have been adjusted to cocircular $\left(\sigma^{+}, \sigma^{+}\right)$as well as linear with relative angles of $0^{\circ}(\uparrow \uparrow), 45^{\circ}(\uparrow \nearrow)$, and $90^{\circ}(\uparrow \rightarrow)$. The first (second) symbol in the parentheses indicates here the polarization of the $\mathbf{k}_{1}\left(\mathbf{k}_{2}\right)$ pulse, respectively. The $1 / e^{2}$ focus diameter of the pulses on the sample was $70 \mu \mathrm{m}$. The FWM signal in the reflected $2 \mathbf{k}_{2}-\mathbf{k}_{1}$ direction was time-integrated and spectrally resolved by a combination of a spectrometer and an optical multichannel analyzer with a spectral resolution of $0.4 \mathrm{meV}$. The samples were kept in a helium cryostat at a temperature of $50 \mathrm{~K}$.

The FWM spectra obtained for different polarization configurations at a negative delay time of $\tau \approx-0.2 \mathrm{ps}\left(\mathbf{k}_{2}\right.$ before $\mathbf{k}_{1}$ ) are shown in Fig. 1. The FWM signal was analyzed in front of the detector by a combination of a $\lambda / 4$ plate and a polarizer. The analyzer polarization is given in the following by the third symbol in the parentheses indicating the configuration. The excitation intensity was $800 \mathrm{~kW} / \mathrm{cm}^{2}$, corresponding to an exciton density of about $2 \times 10^{9} \mathrm{~cm}^{-2}$. The center of the excitation spectrum was set to $2.815 \mathrm{eV}$, in order to avoid continuum contributions but simultaneously excite the first $\left(11 h X_{h}\right)$ and second $(12 h)$ center-of-mass quantized $1 s$ excitons of the heavy hole, and the first of the light hole $\left(11 l X_{l}\right) .^{6,10}$ The coherent excitation of the heavy-

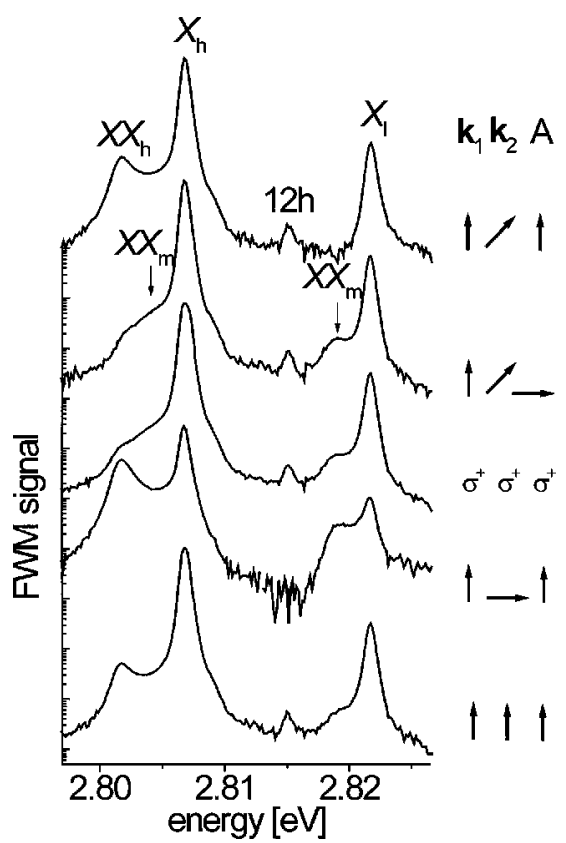

FIG. 1. FWM spectra for different polarization configurations recorded at a delay time of $\tau \approx-0.2 \mathrm{ps}$. The symbols in the parentheses indicate the polarizations of the excitation pulses propagating along $\mathbf{k}_{1}$ and $\mathbf{k}_{2}$ and of the analyzer. 

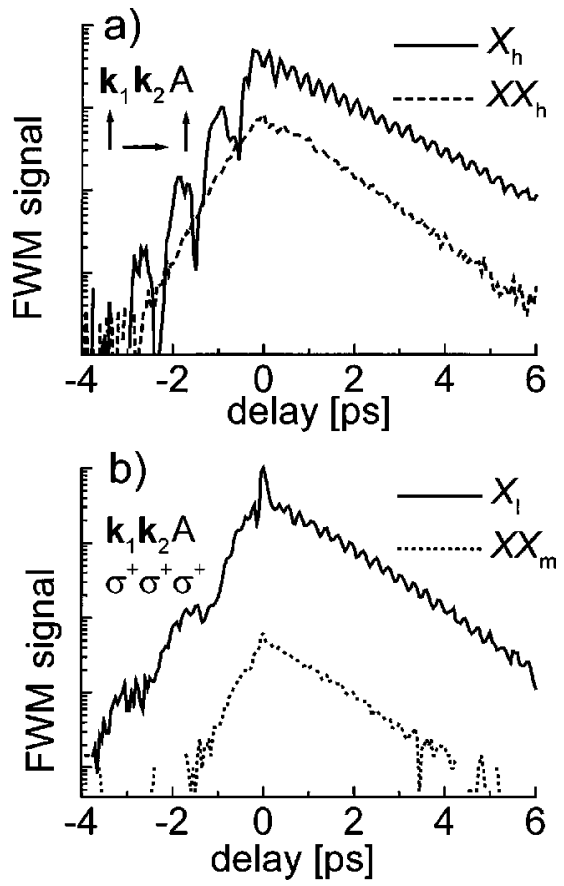

FIG. 2. FWM traces (a) at the heavy-hole $X_{h}$ resonance and the BIF-induced $X X_{h}$ transition for $(\uparrow \rightarrow)$ polarization, and (b) at the light-hole $X_{l}$ resonance and the mixed BIF-induced $X X_{m}$ transition for $\left(\sigma^{+}, \sigma^{+}\right)$polarized fields.

hole $\left(X_{h}\right)$ and light-hole exciton $\left(X_{l}\right)$ states leads to quantum beats in the delay time-dependent FWM signal with a period of $T=270 \mathrm{fs}$ (Fig. 2). The relative phase of the quantum beats is shifted by $\pi$ going from $(\uparrow \uparrow)$ to $(\uparrow \rightarrow)$ excitation, confirming the heavy-hole and light-hole character of the $X_{h}$ and $X_{l}$ exciton transitions, respectively. ${ }^{11} \mathrm{~A}$ significant FWM signal is observed for negative delay times, indicating important interaction-induced FWM processes such as local-field effects (LFE), ${ }^{12}$ excitation-induced dephasing (EID), ${ }^{13,14}$ and biexciton formation (BIF) ${ }^{15-17}$ In homogeneously broadened systems a decay about twice as fast compared to positive delay times is expected for negative delay times for LFE and EID. Inhomogeneous broadening accelerates this decay. The observed FWM signal decay times of 0.5 ps for negative and $1.2 \mathrm{ps}$ for positive delay times indicate the presence of nearly homogeneously broadened exciton resonances.

In addition to the exciton resonances, spectral features in the FWM are observed on the low-energy side of the $X_{h}$ and $X_{l}$ transitions with an energy separation of $\Delta_{h}=4.8 \mathrm{meV}$ and $\Delta_{m}=2.8 \mathrm{meV}$, respectively. The signal close to $\left(X_{h}\right)$, denoted as $\left(X X_{h}\right)$, can be identified as heavy-hole BIF from its polarization dependency, and is also present when exciting only the $\left(X_{h}\right)$ exciton. ${ }^{18}$ The signal on the low-energy side of $\left(X_{l}\right)$ cannot be attributed to a light-hole BIF due to its presence in $\left(\sigma^{+} \sigma^{+} \sigma^{+}\right)$configuration. It is attributed to a heavyhole-light-hole mixed biexciton by the following considerations.

The $X_{h}$ exciton involves a transition from the first heavyhole subband with angular momentum eigenstates $\left|J, J_{z}\right\rangle$ $=\left|\frac{3}{2}, \pm \frac{3}{2}\right\rangle$ to the first electron subband, having angular momentum eigenstates $\left|\frac{1}{2}, \pm \frac{1}{2}\right\rangle$, respectively. The $X_{h}$ eigenstates $\left|J, J_{z}\right\rangle=|1, \pm 1\rangle$ are excited by circularly $\left(\sigma^{ \pm}\right)$polarized light with the dipole matrix vectors $\vec{\mu}_{h}^{ \pm}=-2^{-0.5} \mu_{h}(\mp 1, i)$

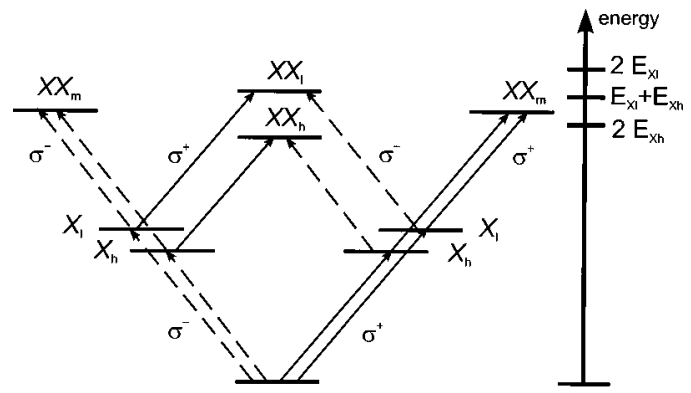

FIG. 3. Energy level diagram indicating the dipole allowed ground-to-exciton and exciton-to-biexciton transitions. The energy scale gives the energetic position of unbound heavy-heavy-hole $X X_{h}$, mixed heavy-light-hole $X X_{m}$, and light-light-hole $X X_{l}$ biexciton states.

in Jones vector notation. Biexcitons are created by a twophoton excitation schematically shown in the energy level diagram of Fig. 3. Bound heavy-hole biexcitons $X X_{h}$ have paired electron as well as hole spins as a consequence of the Pauli exclusion. The $X X_{h}$ two-photon coherence leading to the BIF FWM for negative delay is thus created by a simultaneous $\sigma^{-}$and $\sigma^{+}$excitation by the pulse $\mathbf{k}_{2}$. The thirdorder polarization $P_{2 \mathbf{k}_{2}-\mathbf{k}_{1}}^{(3)}$ generated by pulse $\mathbf{k}_{1}$ from the two-photon coherence gives rise to a time-integrated FWM signal $I_{2 \mathbf{k}_{2}-\mathbf{k}_{1}}^{(3)}(\omega, \tau)$ at the $X_{h}$ transition $\hbar \omega_{h}$ and at the $X_{h}$ $-X X_{h}$ transition $\hbar \omega_{h}-\Delta_{h}$, where $\Delta_{h}$ is the heavy-hole biexciton binding energy. The polarization dependence of the $X X_{h}$ BIF induced FWM is obtained by the solution of optical Bloch equations for a homogeneously broadened many-level system (see Fig. 3), in which the calculated Fourier transformed third-order polarization at $\hbar \omega_{h}-\Delta_{h}$ is proportional to the product of the polarizations of the involved transitions, ${ }^{11}$ i.e.,

$$
\begin{aligned}
P_{2 \mathbf{k}_{2}-\mathbf{k}_{1}}^{(3)}\left(\hbar \omega_{h}-\Delta_{h}\right) \propto & \sum_{\sigma^{+}, \sigma^{-}}\left\{\left[\left(\vec{\mu}_{h}^{+}\right) * \mathbf{E}_{\mathbf{k}_{2}}\right]\left[\left(\vec{\nu}_{h}^{-}\right) * \mathbf{E}_{\mathbf{k}_{2}}\right]\right. \\
& \left.+\left[\left(\vec{\mu}_{h}^{-}\right) * \mathbf{E}_{\mathbf{k}_{2}}\right]\left[\left(\vec{\nu}_{h}^{+}\right) * \mathbf{E}_{\mathbf{k}_{2}}\right]\right\} \\
& \times\left[\left(\vec{\mu}_{h}^{ \pm}\right)^{*} \mathbf{E}_{\mathbf{k}_{1}}\right]^{*} \vec{\nu}_{h}^{\mp} .
\end{aligned}
$$

The $X_{h}$ exciton to $X X_{h}$ biexciton matrix element is given by $\vec{\nu}_{h}^{ \pm}$, and is assumed to be equal to $\vec{\mu}_{h}^{ \pm}$up to a factor close to 1 , since it involves the same interband exciton transition, and the biexciton binding energy is much less than the exciton binding energy. The direction of the BIF-induced third-order polarization is consequently $(\uparrow)$ for $(\uparrow \uparrow),(\uparrow \rightarrow)$, and $(\uparrow \nearrow)$ excitation, which explains the strong reduction of the $X X_{h}$ signal in $(\uparrow \nearrow \rightarrow)$ configuration. The BIF induced signal $X X_{h}$ is equally strong for $(\uparrow \uparrow)$ and $(\uparrow \rightarrow)$ excitation, showing that the BIF process is not affected by EID, as expected for thirdorder processes. It vanishes in $\left(\sigma^{+}, \sigma^{+}\right)$configuration, since the bound biexciton state $X X_{h}$ cannot be excited. The unbound biexciton state ${ }^{5,19,20}$ is neglected for simplicity in the model. It influences in homogeneously broadened systems the signal around the exciton energy, especially for crosslinear polarization, but does not change the signal strength at the bound biexcitonic transitions. The assignment of $X X_{h}$ is further supported by the observation of oscillations in the 
FWM trace at the $X_{h}$ resonance for negative delay times as shown in Fig. 2(a) for $(\uparrow \rightarrow)$ excitation. These oscillations are caused by the interference of LFE and BIF induced polarizations, ${ }^{7}$ and are not present in the $X X_{h}$ trace. From the oscillation period we deduce $\Delta_{h}=4.7 \mathrm{meV}$, in good agreement with $\Delta_{h}=4.8 \mathrm{meV}$ obtained from the FWM spectra.

The FWM trace at the $X_{l}$ resonance for $\left(\sigma^{+}, \sigma^{+}\right)$excitation is displayed in Fig. 2(b). Similar to the behavior at $X_{h}$, the FWM trace at $X_{l}$ shows oscillations according to an energy difference of $2.9 \mathrm{meV}$, in agreement with the $X X_{m}$ energy separation of $\Delta_{m}=2.8 \mathrm{meV}$ from the $X_{l}$ resonance. Again these oscillations do not appear in the trace of $X X_{m}$, and the $X X_{m}$ signal is equally strong in $(\uparrow \uparrow)$ and $(\uparrow \rightarrow)$ configurations, while the $X_{l}$ signal is strongly affected by EID (see Fig. 1). These observations suggest that $X X_{m}$ is caused by BIF, however the attribution to a light-hole biexciton $\left(X X_{l}\right)$ must be excluded since $X X_{m}$ does not vanish for $\left(\sigma^{+}, \sigma^{+}\right)$excitation. This fact implies the formation of mixed heavy-hole-light-hole biexcitons, schematically sketched in Fig. 3. The bound $\left|J, J_{z}\right\rangle=|2, \pm 2\rangle X X_{m}$ biexcitons have different hole spins $\left(\left|\frac{3}{2}, \pm \frac{1}{2}\right\rangle\right.$ and $\left.\left|\frac{3}{2}, \pm \frac{1}{2}\right\rangle\right)$ and opposite electron spins, leading to a polarization dependence of the $X X_{m}$ BIF induced nonlinear polarization at $\hbar \omega_{l}-\Delta_{m}$ according to the product of the involved transitions:

$$
\begin{aligned}
P_{2 \mathbf{k}_{2}-\mathbf{k}_{1}}^{(3)}\left(\hbar \omega_{l}-\Delta_{m}\right) \propto & \sum_{\sigma^{+}, \sigma^{-}}\left\{\left[\left(\vec{\mu}_{h}^{ \pm}\right) * \mathbf{E}_{\mathbf{k}_{2}}\right]\left[\left(\vec{\nu}_{l m}^{ \pm}\right) * \mathbf{E}_{\mathbf{k}_{2}}\right]\right. \\
& \left.+\left[\left(\vec{\mu}_{l}^{ \pm}\right) \mathbf{E}_{\mathbf{k}_{2}}\right]\left[\left(\vec{\nu}_{h m}^{ \pm}\right) * \mathbf{E}_{\mathbf{k}_{2}}\right]\right\} \\
& \times\left[\left(\vec{\mu}_{h}^{ \pm}\right) * \mathbf{E}_{\mathbf{k}_{1}}\right]^{*} \vec{\nu}_{l m}^{ \pm} .
\end{aligned}
$$

Also here, the $X_{l(h)}-X X_{m}$ exciton-biexciton matrix elements $\vec{\nu}_{h m(l m)}^{ \pm}$are assumed to be equal to $\vec{\mu}_{h(l)}^{ \pm}$up to a factor close to unity. The resulting polarization direction of the $X X_{m}$ BIF-induced FWM signal is $\left(\sigma^{+}\right)$for $\left(\sigma^{+} \sigma^{+}\right),(\uparrow)$ for $(\uparrow \uparrow)$, $(\downarrow)$ for $(\uparrow \rightarrow)$, and $(\rightarrow)$ for $(\uparrow \nearrow)$ excitation. Hence the $X X_{m}$ signal appears in $\left(\sigma^{+} \sigma^{+} \sigma^{+}\right)$and $(\uparrow \nearrow \rightarrow)$ configuration while it vanishes in $(\uparrow \nearrow \uparrow)$ configuration, in agreement with the experiment (see Fig. 1). Likewise, the BIF-induced mixed biexciton $X X_{m}$ gives a signal at $\hbar \omega_{h}-\Delta_{m}$ observed in the $\left(\sigma^{+} \sigma^{+} \sigma^{+}\right)$and $(\uparrow \nearrow \longrightarrow)$ configurations.

The relative ratios of the BIF-induced signal intensities can be estimated by comparing the product of oscillator strengths appearing in the third-order nonlinear response. If we assume that the relative oscillator strength between heavy-hole and light-hole related transitions is given by the valence-band functions to $\left|\mu_{h}\right|^{2} /\left|\mu_{l}\right|^{2} \approx\left|\nu_{h}\right|^{2} /\left.\nu_{l}\right|^{2} \approx\left|\nu_{h m}\right|^{2} /\left|\nu_{l m}\right|^{2} \approx 3$, the intensity ratio is approximately given by $I_{X X h}: I_{X X m}: I_{X X l}$ $\approx 81: 9: 1$. The calculated ratio $I_{X X h}: I_{X X m}$ is in good agreement with the experimentally observed ratio of $I_{X X h}: I_{X X m}$ $\approx 10: 1$, and explains why the BIF-induced $X X_{l}$ signal is not visible in the FWM spectra.

In conclusion, we have discussed the FWM responses of nearly homogeneously broadened, quasi-two-dimensional excitons in view of BIF-induced processes. Comparison of polarization-dependent, spectrally resolved FWM with multilevel optical Bloch equations identifies the formation of mixed heavy-light-hole biexcitons $\left(X X_{m}\right)$ appearing at the low-energy side of the heavy-hole $\left(X_{h}\right)$ and light-hole $\left(X_{l}\right)$ exciton resonance. The observed mixed biexciton binding energy $\left(\Delta_{m}=2.8 \mathrm{meV}\right)$ is smaller than the value found for the heavy-heavy biexciton $X X_{h}\left(\Delta_{h}=4.8 \mathrm{meV}\right)$. This is unexpected since the biexciton binding is generally increasing with decreasing electron-hole mass ration, and thus the higher in-plane mass of the light-hole exciton should lead to a higher mixed-biexciton binding energy compared to that of the heavy-hole biexciton. However, there is to our knowledge no theoretical model treating the binding between different kinds of excitons. The estimated intensity ratios of the BIF signals are in agreement with the experimental data, and further explain the missing BIF-induced $X X_{l}$ signal as being too weak to be detected in our experiments.

We thank H. Preis and W. Gebhardt for providing the $\mathrm{Zn}_{0.9} \mathrm{Mg}_{0.1} \mathrm{Se} / \mathrm{ZnSe}$ single QW structure. This work was supported by the Deutsche Forschungsgemeinschaft and by the Danish Ministries of Research and Education in the framework of the Center for Nanostructures.
*Author to whom correspondence should be addressed. Present address: Universität Regensburg-Physik II, Universitätsstraße 31, D-93040 Regensburg, Germany. FAX: 49 9419434226. Electronic address: Hans-Peter.Wagner@physik.uni-regensburg.de (or) HP.Wagner@physik.tu-chemnitz.de

${ }^{1}$ Q. Fu, D. Lee, A. Mysyrowicz, A. V. Nurmikko, R. L. Gunshor, and L. A. Kolodziejeski, Phys. Rev. B 37, 8791 (1988).

${ }^{2}$ Y. Yamada, T. Mishima, Y. Masumoto, Y. Kawakami, J. Suda, S. Fujita, and S. Fujita, Phys. Rev. B 52, R2289 (1995).

${ }^{3}$ F. Kreller, M. Lowisch, J. Puls, and F. Henneberger, Phys. Rev. Lett. 75, 2420 (1995).

${ }^{4}$ V. Kozlov, P. Kelkar, A. V. Nurmikko, C.-C. Chu, D. C. Grillo, J. Han, C. G. Hua, and R. L. Gunshor, Phys. Rev. B 53, 10837 (1996).

${ }^{5}$ T. Häupl, N. Nikolaus, F. Henneberger, and A. Schülzgen, Phys. Status Solidi B 194, 219 (1996)

${ }^{6}$ T. Kuroda, K. Inoue, R. Kuribayashi, F. Minami, A. Mysyrowicz, and I. Suemune, J. Lumin. 66\&67, 429 (1996).

${ }^{7}$ H. P. Wagner, R. Maier, A. Schätz, W. Langbein, and J. M.
Hvam, Phys. Rev. B 57, 1791 (1998).

${ }^{8}$ D. Birkedal, J. Singh, V. G. Lyssenko, J. Erland, and J. M. Hvam, Phys. Rev. Lett. 76, 672 (1996).

${ }^{9}$ M. Wörz, E. Griebl, Th. Reisinger, B. Flierl, D. Haserer, T. Semmler, T. Frey, and W. Gebhardt, Phys. Status Solidi B 202, 805 (1997).

${ }^{10}$ H. Mathieu, P. Lefebvre, and P. Christol, Phys. Rev. B 46, 4092 (1992).

${ }^{11}$ K. Bott, E. J. Mayer, G. O. Smith, V. Heuckeroth, M. Hübner, J. Kuhl, T. Meier, A. Schulze, M. Lindberg, S. W. Koch, P. Thomas, R. Hey, and K. Ploog, J. Opt. Soc. Am. B 13, 1026 (1996).

${ }^{12}$ M. Wegener, D. S. Chemla, S. Schmitt-Rink, and W. Schäfer, Phys. Rev. A 42, 5675 (1990).

${ }^{13}$ H. Wang, K. Ferrio, D. G. Steel, Y. Z. Hu, R. Binder, and S. W. Koch, Phys. Rev. Lett. 71, 1261 (1993).

${ }^{14}$ H. Wang, K. B. Ferrio, D. G. Steel, P. R. Berman, Y. Z. Hu, R. Binder, and S. W. Koch, Phys. Rev. A 49, R1551 (1994).

${ }^{15}$ K. Bott, O. Heller, D. Bennhardt, S. T. Cundiff, P. Thomas, E. J. Mayer, G. O. Smith, R. Eccleston, J. Kuhl, and K. Ploog, Phys. Rev. B 48, 17418 (1993). 
${ }^{16}$ H. Wang, J. Shah, T. C. Damen, and L. N. Pfeiffer, Solid State Commun. 91, 869 (1994).

${ }^{17}$ Y. Z. Hu, R. Binder, S. W. Koch, S. T. Cundiff, H. Wang, and D. G. Steel, Phys. Rev. B 49, 14382 (1994).

${ }^{18}$ H. P. Wagner, A. Schätz, R. Maier, W. Langbein, and J. M. Hvam, Physica E 2, 82 (1998).
${ }^{19}$ A. E. Paul, J. A. Bolger, A. L. Smirl, and J. G. Pelegrino, J. Opt. Soc. Am. B 13, 1016 (1996).

${ }^{20}$ T. F. Albrecht, K. Bott, T. Meier, A. Schulze, M. Koch, S. T. Cundiff, J. Feldmann, W. Stolz, P. Thomas, S. W. Koch, and E. O. Göbel, Phys. Rev. B 54, 4436 (1996). 